\title{
Implementation of the Nursing Process in Naivasha District Hospital, Kenya
}

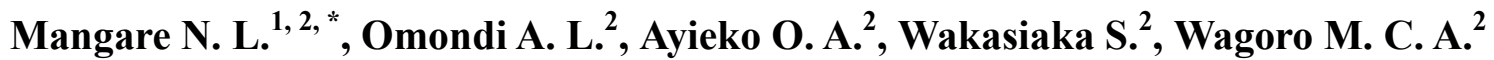 \\ ${ }^{1}$ Department of Nursing, Kisii University, Kisii, Kenya \\ ${ }^{2}$ School of Nursing, University of Nairobi, Nairobi, Kenya
}

Email address:

lnyatichi@kisiiuniversity.ac.ke (Mangare N. L.), liliathis@gmail.com (Omondi A. L.), ayieko@uonbi.ac.ke (Ayieko O. A.), swakasiaka@gmail.com (Wakasiaka S.), carole@uonbi.ac.ke (Wagoro M. C. A.)

${ }^{*}$ Corresponding author

\section{To cite this article:}

Mangare N. L., Omondi A. L., Ayieko O. A., Wakasiaka S., Wagoro M. C. A. Implementation of the Nursing Process in Naivasha District Hospital, Kenya. American Journal of Nursing Science. Vol. 5, No. 4, 2016, pp. 152-157. doi: 10.11648/j.ajns.20160504.15

Received: July 6, 2016; Accepted: July 15, 2016; Published: August 3, 2016

\begin{abstract}
The Nursing Process (NP) is a systematic rational method of planning and providing individualized quality Nursing Care (QNC). Training curricula for nurses and midwives in Kenya have incorporated the NP as a framework for nursing care. In addition, many institutions in Kenya have initiated continuous education to nurses on the nursing process. However, there are no studies indicating the level of utilization of this framework in Kenyan health institutions. This study was aimed at establishing the implementation of the NP in Naivasha District Hospital. This was a cross sectional study conducted on 83 nurses and midwives selected using quota sampling. A structured questionnaire and an observation checklist was used to collect data then analyzed using the statistical package for social sciences (SPSS). Descriptive statistics were used to summarize the data and Pearson's Correlation Coefficient was used to establish correlation between variables. The nursing process has been initiated in Naivasha District Hospital but nurses use it in an incomplete and unsystematic manner. Nurses in this hospital have a positive attitude towards the NP but have difficulties performing all the phases of the NP.
\end{abstract}

Keywords: Nursing Process, Quality Nursing Care, Implementation

\section{Introduction}

The NP was first described by the nurse theorist Ida Jean Orlando in her theory 'Deliberative Nursing Process' [1]. It is a scientific and dynamic method used to deliver quality nursing care. It's made up of five interrelated steps: assessment, diagnosis, planning, implementation and evaluation [2]. It facilitates progress in the nursing profession, research and eases management [3].

The quality of nurses' work improves when they use the NP, which ultimately positively affects patients' outcomes during and after hospitalization [4]. Failure to use the NP leads to low QHC, disorganization of the nursing care and conflicting roles [5].

Majority of nurses have knowledge of the nursing process, but they do not apply it in practice. [5]. A few nurses carry out tasks related to the various stages of the nursing process (NP), but do not implement it systematically. Nurses also report difficulties in implementing all the phases of this framework [6-8].

Some nurses report that this framework should be used by students to pass examinations and for providing nursing care for critically ill patients only [9]. Although the NP is seen as a form of professional recognition with regard to its role in society and something that allows nurses to have authenticity and freedom of action in their practice, it is also viewed with feelings of anger, dissatisfaction, and frustration [10].

\section{Theoretical Framework}

This study is based on the Theory of Planned Behavior which suggests that behavior is dependent on one's intention to perform the behavior. Intention is determined by an individual's attitude (beliefs and values about the outcome of the behavior) and subjective norms (beliefs about what other people think the person should do or general social 
pressure). Behavior is also determined by an individual's perceived behavioral control which is an individual's perception of their ability or feelings of self-efficacy to perform the behavior [11].

\subsection{Statement of the Problem}

The quality of health services in Kenyan public health institutions is low and patients are dissatisfied with nursing services $[12,13]$. Nurses make up $51.5 \%$ of all health workers in Kenyan public hospitals [14]. This implies that nursing services contribute a large percentage towards overall healthcare. Therefore in order to improve the overall quality of healthcare, nursing services must be improved.

The quality of nursing care improves when the nurses use the NP [4]. This therefore implies that one main strategy of improving the QNC is through the use of the NP. This framework is incorporated into all the nursing curricula in Kenya. However, reports at the CNO's office at the Ministry of Health indicate that in most hospitals this framework is not used in the provision of nursing care and if used, the phases are not systematically followed [15]. It is due to this that a process of mainstreaming the nursing process in clinical settings in Kenya was initiated. Naivasha District hospital is one of the institutions that has benefited from this training. This study was therefore set to establish implementation of the NP in this hospital.

\subsection{Aims}

The broad objective was to establish the implementation of the nursing process by nurses working in NDH. The specific objectives were:

i. To establish the implementation of the NP.

ii. To determine the attitude of the nurses towards the NP.

iii. To establish the phases of the NP nurses find difficult to perform.

\section{Methodology}

This was a quantitative cross sectional study on 83 nurses. The study was approved by Kenyatta National Hospital/ University of Nairobi Research and Ethics Committee (KNH/ UON ERC) Ref: KNH-ERC/A124. The sample size was determined using the Cochran's formulae [16]. The sample was selected using quota sampling. The quotas included pediatric, male and female wards, theatre, maternity, neonatal unit, outpatient and MCH/FP clinics. Purposive sampling was then used to select the required sample from each quota. A list of nurses working in each department was obtained from the nursing officer in-charge which was used as the sampling frame. A structured questionnaire and an observation checklist were used to collect data. The data was entered in Microsoft excel computer software and analyzed using the statistical package for social sciences (SPSS) version 20.0. Pearson's Correlation Coefficient was used to establish correlation between variables.

\section{Results}

\subsection{Implementation of the Nursing Process}

A checklist was used to observe use of the nursing process by each of the participants on one patient. The checklist had items on work environment, comprehensive assessment, completion of patient charts and utilization of the nursing care plan as given below.

\subsubsection{Work Environment}

All the floors of the participants' work stations, patients, patient lockers or incubators and linen were clean. Thirty one (37.3\%) participants' patients did not require lockers or incubators, five patients $(6 \%)$ did not have linen while eighteen $(21.7 \%)$ participants' patients did not require linen. Forty five $(54.2 \%)$ of the participants' patients did not have caretakers in the ward or clinic while thirty one $(37.3 \%)$ had caretakers as shown in Table 1 below.

\subsubsection{Comprehensive Assessment}

All the participants had identification data on the patients' notes. Majority of the participants $(77.1 \%, \mathrm{n}=64)$ assessed the chief complaint and $48.2 \% \quad(n=40)$ of the participants assessed the history of presenting illness. Most of the participants did not assess the socioeconomic $(57.8 \%, \mathrm{n}=48)$, family history $(61.4 \%, \mathrm{n}=51)$ and physical examination $(61.4 \%, n=51)$. None of the participants assessed the current health state, allergies and treatments. A summary of investigations done and ordered was assessed by only $39.8 \%$ $(n=33)$ of the participants. Thirteen participants $(15.7 \%)$ were not required to assess the chief complaint, history of presenting illness, socioeconomic history, and history of past illness and family history as shown in Table 1 below.

\subsubsection{Completion of Patient Charts}

Sixty seven participants $(80.7 \%)$ had identification data on the patients charts, 43 participants $(51.8 \%)$ did not complete the TPR \& BP charts appropriately while $33.7 \% \quad(n=28)$ failed to complete the fluid charts appropriately. However majority of the other charts (treatment, transfusion, feeding, CWC card, maternal card and partogram) were appropriately completed. Fourteen participants (16.9\%), 46 (55.4\%) and 17 (20.5\%) were not required to complete TPR\& BP, fluid and other charts respectively as shown in Table 1.

Table 1. Work environment, comprehensive assessment and completion of patient charts.

\begin{tabular}{|c|c|c|c|}
\hline Item & Yes & No & Not applicable \\
\hline \multicolumn{4}{|l|}{ Work environment } \\
\hline Floors cleanliness & $83(100 \%)$ & $0(0 \%)$ & $0(0 \%)$ \\
\hline
\end{tabular}




\begin{tabular}{llll}
\hline Item & Yes & No & Not applicable \\
\hline Hygiene of patient lockers/ incubators & $52(62.7 \%)$ & $0(0 \%)$ & $31(37.3 \%)$ \\
Availability of patient linen & $60(72.3 \%)$ & $5(6 \%)$ & $18(21.7 \%)$ \\
Hygiene of patient linen & $60(72.3 \%)$ & $0(0 \%)$ & $23(24.1)$ \\
Presence of caretakers & $31(37.3 \%)$ & $45(54.2 \%)$ & $7(27.3 \%)$ \\
Comprehensive assessment & & & \\
Identification data & $83(100 \%)$ & $0(0 \%)$ & $0(0 \%)$ \\
Chief complaint & $64(77.1 \%)$ & $6(7.2 \%)$ & $13(15.7 \%)$ \\
History of presenting illness & $40(48.2 \%)$ & $30(36.1 \%)$ & $13(15.7 \%)$ \\
History of past illness & $26(31.3 \%)$ & $44(53 \%)$ & $13(15.7 \%)$ \\
Socioeconomic history & $22(26.5 \%)$ & $48(57.8 \%)$ & $13(15.7 \%)$ \\
Family history & $19(22.9 \%)$ & $51(61.4 \%)$ & $13(15.7 \%)$ \\
Current health state/ allergies and treatments & $0(0 \%)$ & $83(100 \%)$ & $0(0 \%)$ \\
Data on physical examination & $32(38.6 \%)$ & $51(61.4 \%)$ & $0(0 \%)$ \\
Summary of investigations done and ordered & $33(39.8 \%)$ & $37(44.6 \%)$ & $13(15.7 \%)$ \\
Appropriate completion of patient charts & & & \\
Identification data & $67(80.7 \%)$ & $16(19.3 \%)$ & $0(0 \%)$ \\
TPR \& BP charts & $26(31.3 \%)$ & $43(51.8 \%)$ & $14(16.9 \%)$ \\
Fluid charts & $9(10.8 \%$ & $28(33.7 \%)$ & $46(55.4 \%)$ \\
Others (treatment, transfusion, feeding, CWC card, maternal card, partogram) & $47(56.6 \%)$ & $19(22.9 \%)$ & $17(20.5 \%)$ \\
\hline
\end{tabular}

\subsection{Utilization of the Nursing Care Plan}

According to the study, the proportion of the participants who carried out the various components of the nursing care plan appropriately ranged between $15.7 \%$ and $30.1 \%$ as shown in Table 2 below.

Table 2. Utilization of the nursing care plan.

\begin{tabular}{llll}
\hline Item & Yes & No & Not applicable \\
\hline 3 part nursing diagnosis used & $25(30.1 \%)$ & $58(69.9 \%)$ & $0(0 \%)$ \\
Nursing diagnosis addresses health problems holistically (physiological, psychological, spiritual, social) & $19(22.9 \%)$ & $64(77.1 \%)$ & $0(0 \%)$ \\
Organized in order of priority & $21(25.3 \%)$ & $62(74.7 \%)$ & $0(0 \%)$ \\
Client oriented goal & $25(30.1 \%)$ & $58(69.9 \%)$ & $0(0 \%)$ \\
Goal relevant to nursing diagnosis & $25(30.1 \%)$ & $58(69.9 \%)$ & $0(0 \%)$ \\
Plan of action Consistent with the respective goal & $24(28.9 \%)$ & $59(28.9 \%)$ & $0(0 \%)$ \\
Plan of action Arranged in order of priority & $21(25.3 \%)$ & $62(74.7 \%)$ & $0(0 \%)$ \\
Plan of action Safe for patients & $25(30.1 \%)$ & $58(69.9 \%)$ & $0(0 \%)$ \\
Rationale indicated for each nursing intervention & $24(28.9 \%)$ & $59(71.1 \%)$ & $0(0 \%)$ \\
Rationale relevant to the nursing intervention & $24(28.9 \%)$ & $59(71.1 \%)$ & $0(0 \%)$ \\
Indicates the extent to which the outcome criteria has been achieved (evaluation) & $13(15.7 \%)$ & $70(84.3 \%)$ & $0(0 \%)$ \\
Accurate documentation is done in the charts and nursing care plan & $13(15.7 \%)$ & $70(84.3 \%)$ & $0(0 \%)$ \\
Records contain name and signature of the nurse & $67(19.3 \%)$ & $16(19.3 \%)$ & $0(0 \%)$ \\
\hline
\end{tabular}

\subsection{Attitude of Nurses Towards the Nursing Process}

Nine statements were used to elicit the attitude of nurses towards the NP through a five point likert scale. Forthy three participants $(51.8 \%)$ strongly agreed that the nursing process facilitates total patient care while $32.7 \%(\mathrm{n}=27)$ agreed.

Thirty six participants (43.4\%) strongly disagreed that nursing care plans are a waste of time because nobody looks at them and $19(22.9 \%)$ agreed. Majority of the nurses $(86.7 \%)$ stronlgy agreed that setting goals enables meaningful evaluation of care.

The proportions of participants who strongly agreed and agreed that patient care should have less writing and more action were $28.9 \%$ and $27.7 \%$ respectively. Fifty nine nurses (71.1\%) strongly disagreed that a nursing care plan is not significant in provision and evaluation of care. Majority of the participants $(60.2 \%)$ strongly disagreed that nursing care plans are a mere academic exercise while $87.7 \%$ strongly agreed that care plans facilitate establishment of priorities of care. The proportions of participants who strongly agreed and agreed that patients should be involved in their plan of care were $48.2 \%$ and $25.3 \%$ respectively. Twenty eight participants $(33.7 \%)$ strongly agreed that nursing care plans have no relation to what the nurse actually implements while $19(22.9 \%)$ strongly agreed. Overall, majority of the nurses attitude towards the nursing process was positive (Table 3 ). 
Table 3. Participants' Responses to Various Statements on Attitude.

\begin{tabular}{lllllll}
\hline Statement & SD=1 N (\%) & $\mathbf{D = 2} \mathbf{~ N ~ ( \% )}$ & $\mathbf{U C = 3} \mathbf{~ N}(\%)$ & $\mathbf{A = 4} \mathbf{~ N}(\%)$ & SA=5 N (\%) & Mean \\
\hline $\begin{array}{l}\text { I find using nursing care plans facilitating total patient care } \\
\text { rather than concentrating on tasks. }\end{array}$ & $6(7.2 \%)$ & $1(1.2 \%)$ & $6(7.2 \%)$ & $27(32.5 \%)$ & $43(51.8 \%)$ & 4.20 \\
$\begin{array}{l}\text { I don't mind writing care plans but it is a waste of time } \\
\text { because nobody looks at them. }\end{array}$ & $36(43.4 \%)$ & $19(22.9 \%)$ & $8(9.6 \%)$ & $11(13.3 \%)$ & $910.8 \%)$ & 2.25 \\
$\begin{array}{l}\text { Setting realistic patient goals enables the nurse to make a } \\
\text { meaningful evaluation of care. }\end{array}$ & 0 & 0 & 0 & $11(13.3 \%)$ & $72(86.7 \%)$ & 4.87 \\
$\begin{array}{l}\text { In patient care there should be less writing and more } \\
\text { action. }\end{array}$ & $12(14.5 \%)$ & $15(18.1 \%)$ & $9(10.8 \%)$ & $23(27.7 \%)$ & $24(28.9)$ & 3.39 \\
$\begin{array}{l}\text { The care plan plays no significant part in the provision and } \\
\text { evaluation of care. }\end{array}$ & $59(71.1 \%)$ & $13(15.7 \%)$ & $2(2.4 \%)$ & $4(4.8 \%)$ & $5(6 \%)$ & 1.59 \\
$\begin{array}{l}\text { I think writing nursing care plans is merely an academic } \\
\text { exercise. }\end{array}$ & $50(60.2 \%)$ & $16(19.3 \%)$ & $4(4.8 \%)$ & $4(4.8 \%)$ & $9(10.8 \%)$ & 1.87 \\
$\begin{array}{l}\text { Use of nursing care plans enables a nurse to establish } \\
\text { priorities of care. }\end{array}$ & $5(6 \%)$ & 0 & $6(7.2 \%)$ & 0 & $72(86.7 \%)$ & 4.69 \\
$\begin{array}{l}\text { I believe care plans should be discussed with the patient, so } \\
\text { that the nurse can learn how he feels about his problems. }\end{array}$ & $6(7.2 \%)$ & $8(9.6 \%)$ & $8(9.6 \%)$ & $21(25.3 \%)$ & $40(48.2 \%)$ & 3.98 \\
$\begin{array}{l}\text { I find the nursing instructions on a care plan often bear } \\
\text { very little relation to what the nurse actually does. }\end{array}$ & $28(33.7 \%)$ & $17(20.5 \%)$ & $7(8.4 \%)$ & $12(14.5 \%)$ & $19(22.9 \%)$ & 2.72 \\
\hline
\end{tabular}

Key: $\mathrm{SD}=$ strongly disagree, $\mathrm{D}=$ disagree, $\mathrm{UC}=$ uncertain, $\mathrm{A}=$ agree, $\mathrm{SA}=$ strongly agree

\subsection{Correlation Between Demographic Characteristics and Various Statements Eliciting Attitude}

Gender had a statistically significant correlation with the fourth statement, 'in patient care there should be less writing and more action' $(\mathrm{r}=-0.284, \mathrm{p}=0.009, \mathrm{n}=83)$ and ninth statement 'I find the nursing instructions on a care plan often bear very little relation to what the nurse actually does' $(r=-0.256, p=0.020, n=83)$. The rest of the statements had statistically insignificant correlations as shown in Table 4.

Table 4. Correlation between Demographic Characteristics and Various Statements Eliciting Attitude.

\begin{tabular}{|c|c|c|c|c|c|c|c|c|c|c|}
\hline Variables & Statistical Test & Attitude 1 & Attitude 2 & Attitude 3 & Attitude 4 & Attitude 5 & Attitude 6 & Attitude 7 & Attitude 8 & Attitude 9 \\
\hline \multirow{2}{*}{$\begin{array}{l}\text { Professional } \\
\text { qualification }\end{array}$} & Pearson Correlation & -.081 & -.094 & -.122 & -.089 & -.120 & -.095 & -.080 & -.015 & .098 \\
\hline & P-value & .469 & .398 & .271 & .426 & .278 & .391 & .475 & .895 & .377 \\
\hline \multirow{2}{*}{ Gender } & Pearson Correlation & .075 & -.201 & -.092 & $-.284^{* *}$ & -.079 & -.023 & .073 & .007 & $-.256^{*}$ \\
\hline & P-value & .501 & .069 & .407 & .009 & .480 & .834 & .513 & .953 & .020 \\
\hline \multirow{2}{*}{ Age } & Pearson Correlation & .039 & .036 & -.096 & .070 & -.055 & .098 & -.041 & -.210 & .141 \\
\hline & P-value & .727 & .746 & .389 & .531 & .624 & .377 & .715 & .057 & .203 \\
\hline \multirow{2}{*}{$\begin{array}{l}\text { Years of Clinical } \\
\text { experience }\end{array}$} & Pearson Correlation & -.111 & .083 & -.168 & .027 & -.093 & .063 & -.037 & -.175 & .080 \\
\hline & P-value & .320 & .458 & .129 & .807 & .405 & .574 & .738 & .114 & .471 \\
\hline \multirow{2}{*}{$\begin{array}{l}\text { Current work } \\
\text { station }\end{array}$} & Pearson Correlation & .026 & -.063 & -.138 & -.043 & -.017 & -.040 & .064 & -.148 & .136 \\
\hline & P-value & .817 & .570 & .212 & .702 & .878 & .722 & .566 & .181 & .219 \\
\hline
\end{tabular}

*Correlation is significant at the 0.05 level. Attitude 1-9=statements 1-9 used to elicit attitude.

\subsection{Phases of the NP Posing Difficulties to Participants}

Participants were asked to rate how difficult or easy they found various phases of the NP using a five point Likert scale. Overall the participants found all the phases easy to perform as given in the Table 5 below.

Table 5. Phases of the NP Posing Difficulties to Participants.

\begin{tabular}{lllllll}
\hline Phase & Very easy & Easy & Uncertain & Difficult & Very Difficult & Total \\
\hline Assessment & $18.1 \%$ & $62.7 \%$ & $4.8 \%$ & $7.2 \%$ & $7.2 \%$ & $100 \%$ \\
Diagnosing & $13.3 \%$ & $44.6 \%$ & $12 \%$ & $25.3 \%$ & $4.8 \%$ & $100 \%$ \\
Goal identification & $13.3 \%$ & $57.8 \%$ & $13.3 \%$ & $12 \%$ & $3.6 \%$ & $100 \%$ \\
Planning & $19.3 \%$ & $55.4 \%$ & $9.6 \%$ & $10.8 \%$ & $4.8 \%$ & $100 \%$ \\
Implementing & $16.9 \%$ & $43.4 \%$ & $15.7 \%$ & $16.9 \%$ & $7.2 \%$ & $100 \%$ \\
Evaluating & $14.5 \%$ & $54.2 \%$ & $12 \%$ & $13.3 \%$ & $6 \%$ & $100 \%$ \\
Documenting & $21.7 \%$ & $49.4 \%$ & $9.6 \%$ & $13.3 \%$ & $6 \%$ & $100 \%$ \\
\hline
\end{tabular}




\section{Discussion}

Majority of the nurses in the study had a diploma in nursing; this may be attributed to the nursing council of Kenya's efforts to ensure all the nurses in the country have at least a diploma and above in order to improve the quality of nursing care [17]. This also implies that most of the nurses in this hospital are capable of adopting the NP in the delivery of nursing care. The age distribution of participants was varied ranging from 25 to 60 years; this is because nurses are employed at different ages.

From the observation made during the study, all participants had identification data on patients' notes. Majority of the participants $(77.1 \%, n=64)$ assessed the main complaint and $48.2 \% \quad(n=40)$ assessed the history of presenting illness. However, most participants did not assess socioeconomic, family history and physical examination. None of the participants assessed the current health state, allergies and treatments. A summary of investigations done and ordered was assessed by only $39.8 \%(n=33)$ of the participants. This implies that majority of participants did not carry out a comprehensive assessment of the patients. This contradicts the finding above in which majority of participants reported that they find assessment phase of the NP easy.

Sixty seven participants $(80.7 \%)$ had identification data on patients' charts, 43 participants (51.8\%) did not complete the TPR \& BP charts appropriately while $33.7 \%(n=28)$ failed to complete fluid charts appropriately. However majority of the other charts (treatment, transfusion, feeding, CWC card, maternal card and partogram) were appropriately completed. This implies that participants had a challenge in completing fluid and TPR \& BP charts which also contradicts the findings above in which participants reported to find documentation easy.

The largest proportion of participants did not carry out various components of the nursing care plan appropriately. This could be attributed to inadequate time, inadequate knowledge, nursing diagnosis terminologies, lack of experience on using the NP, lack of policies, lack of reference materials, lack of incentives, inadequate supplies, inadequate staffing and lack of printed assessment forms. These results are similar to those previous studies in which nurses were reported to have difficulties in performing all the phases of the NP [7].

Most participants agreed that the NP facilitates total patient care, nursing care plans are not mere administrative tasks, setting realistic patient goals enables the nurse to make a meaningful evaluation of care, patients should be involved in their plan of care and that in-patient care there should be less writing and more action. They however disagreed that the care plan plays no significant part in provision and evaluation of care and that writing nursing care plans is merely an academic exercise. This therefore means these participants have a positive attitude towards the nursing process. However, there is a discrepancy between their attitude and their low use of the NP as observed.

Most participants reported that they find assessment, formulating nursing diagnoses, setting goals of care, implementing the plan, evaluating the care and documenting easy. However, there is a discrepancy between these findings and the very low proportion of participants who appropriately carry out these phases in actual patient care. These findings are similar to those from previous studies which showed that formulation of the nursing diagnoses was a challenge for nurses [6-8].

\section{Conclusion}

(1) The nursing process (NP) has been initiated in Naivasha district hospital (NDH) but nurses use it in an incomplete and unsystematic manner.

(2) Nurses in NDH have a positive attitude towards the NP.

(3) Participants however have difficulties performing all the phases of the NP (assessment, diagnosing, goal identification, planning, implementing, evaluation and documentation) although they report that they find all of them easy.

\section{Recommendations}

(1) The nurses should use the available resources to improve implementation of the nursing process.

(2) Continuous professional development sessions on the nursing process should be strengthened in order to improve the level of knowledge of the nurses in the hospital.

(3) Supportive supervision on the use of the NP should be strengthened.

(4) A policy on use of the NP in patient care should be implemented.

(5) A similar study should be done in the Kenya.

\section{Acknowledgement}

The project described was supported by the LinkedStrengthening Maternal, Newborn and Child Health Research Training in Kenya through a grant from the US National Institutes of Health (Number 5R24TW008907). The content is solely the responsibility of the authors and does not necessarily represent the official views of the US National Institutes of Health.

\section{References}

[1] Basavanthappa B. T. (2007). Nursing Theories. First edition. New Delhi, India: Jaypee.

[2] Alfaro-LeFevre, R. (2010). Applying nursing process: A tool for critical thinking ( $7^{\text {th }}$ Ed.). Philadelphia: Lippincott, Williams \& Wilkins. 
[3] Urquhart C., Currell R., Grant M. J., et al. (2009). Nursing record systems: effects on nursing practice and healthcare outcomes. Cochrane Database of Systematic Reviews, 1, CD002099. Available from: www.ncbi.Nlm.nih.gov /pubmed/19160206. Retrieved on $15^{\text {th }}$ January, 2012.

[4] Lee J. L., Chang B. L., Pearson M. L., et al. (1999). Does what nurses do affect clinical outcomes for hospitalized patients? A review of the literature. Health Services Research, 34 (5), 1011-1032.

[5] Andrade J. S., Vieira M. J. (2005). Nursing care in practice: Problems, perspectives, and need for systematization. Revista Brasileira de Enfermagem, 58 (3), 261-265.

[6] Granero-Molina J., Fernández-Sola C., Aguilera-Manrique G. et al. (2010). Analysis of conditions for introducing nursing procedures in Santa Cruz (Bolivia). Enfermería Clínica, 20 (5), 280-285.

[7] Lima A. F. \& Kurcgant P. (2006a). The nursing diagnosis implementation process at the university hospital of the University of São Paulo. Revista da Escola de Enfermagem da U S P, 40 (1), 111-116.

[8] Lee T. T. (2005). Nursing diagnoses: Factors affecting their use in charting standardized care plans. Journal of Clinical Nursing, 14, 640-647.

[9] Gatere, G. (1993). A study on use of nursing care plans. Kenya Journal of Nursing, 1, 15-19.

[10] Alvez A. R., Lopes C. H., Jorge M. (2008). The meaning of the nursing process for nursing of intensive therapy units: An interactionist approach. Revista da Escola de Enfermagem da US P, 42 (4), 649-655.

[11] Ajzen I. (1991). The theory of planned behavior. Organizational Behavior and Human Decision Processes, 50, 179-211.

[12] Ojwang, B. O., Ogutu, E. A., \& Matu, P. M. (2010). Nurses' impoliteness as an impediment to patients' rights in selected Kenyan hospitals. International Journal of Health and Human Rights, 12 (2).

[13] Kenya National Commission on Human Rights (2011). Silenced minds: The systematic neglect of the mental health system in Kenya. Available from: $<$ http://www.knchr.org/Portals/0/Reports/THE_\%20MENTAL _HEALTH_REPORT.pdf $>$ Retrieved on April, 2012.

[14] National Human Resources for Health (HRH) annual Report. (2010). Available from: http://www.capacitykenya.org. Retrieved on 25+ September 2012.

[15] Ministry of Health (MoH) (2010). Kenya's Health sector satisfaction survey report, Nairobi, Kenya.

[16] Institute of Food and Agricultural Sciences (IFAS), (2009). Determining Sample size. Available from: http://edis.ifas.ufl.edu. Retrieved on $18^{\text {th }}$ January, 2012.

[17] Rakuom C. (2010). Nursing human resources in Kenya. International Centre for Human Resources in Nursing 3, 2829. 